\title{
Percepções sobre o Poder dos Dirigentes Máximos dos Serviços do Ministério da Justiça em Portugal: evidências estatísticas de diferenças em função do gênero dos colaboradores?
}

\author{
Perceptions on Portuguese Ministry of Justice Agencies Leaders' Power: \\ statistical evidence of gender differences among employees?
}

\author{
Pedro Miguel Alves Ribeiro Correia \\ Universidade de Lisboa, Lisboa - Portugal
}

Resumo: Neste artigo são apresentados os resultados de um estudo sobre as percepções dos colaboradores dos serviços do Ministério da Justiça em Portugal acerca do poder detido pelo dirigente máximo do serviço ao qual pertencem. É dada particular atenção à temática do gênero, utilizando a dicotomia homem-mulher como caso particular da componente disposicional das perceções. Foi realizado um inquérito por meio de questionário com 458 colaboradores dos serviços do Ministério da Justiça português. Os resultados obtidos evidenciam estatisticamente que as perceções sobre o poder detido pelo dirigente máximo do serviço, em que cada participante no estudo desenvolve a sua atividade laboral, apresentam diferenças em função do gênero.

Palavras-chave: Ministério da Justiça em Portugal. Poder dos Dirigentes. Diferenças em Função do Gênero.
Abstract: This article presents the results of a study on the perceptions of Portuguese Ministry of Justice agencies employees regarding the power held by the leader of the agency to which they belong. Particular attention is given to the issue of gender, using the dichotomy between men and women as a particular case of the dispositional component of perceptions. A survey of 458 employees of the Portuguese Ministry of Justice agencies was conducted. The results statistically show that the perceptions about the power held by the leader of the agency, where each study participant works, differ according to gender.

Keywords: Portuguese Ministry of Justice. Leader's Power. Gender Differences.

Recebido em: 26/12/2015

Revisado em: 20/11/2016

Aprovado em: 05/12/2016 


\section{Introdução}

Em Portugal, em 2008, com a entrada em vigor da Lei n. 66B/2007, de 28 de dezembro de 2007, que institui o Sistema Integrado de Gestão e Avaliação do Desempenho da Administração Pública (SIADAP) como instrumento de avaliação de desempenho na administração pública, ocorre um reacender do interesse e uma intensificação do debate político, académico e público sobre as ideias que devem pautar e governar a atuação dos dirigentes máximos das organizações públicas.

Essa gestão integrada do desempenho tem decorrido num ciclo de gestão no qual, após serem calendarizados e fixados de forma quantitativa os objetivos de desempenho dos programas de atividades, o desempenho efetivo é medido e reportado. Tal definição quantitativa e calendarizada de objetivos para o ano seguinte tem tido em conta, em maior ou menor extensão consoante o grau de sofisticação da gestão do serviço em causa, a missão do serviço, as suas atribuições, os objetivos estratégicos plurianuais determinados superiormente, os resultados da avaliação do desempenho, as disponibilidades orçamentais, os respetivos mapas de pessoal e, com particular relevo para o presente artigo, os compromissos assumidos na carta de missão pelo dirigente máximo de cada organização.

A teoria institucional e o conceito de ranking podem ajudar na compreensão da forma como os dirigentes máximos dos serviços do Ministério da Justiça implementam e interpretam o SIADAP (CORREIA, 2011; 2012), mas ajudam igualmente a entender de forma mais clara as perceções desenvolvidas pelos colaboradores que têm a seu cargo, face ao exercício do poder institucional de que são detentores. Contudo, a teoria institucional, exclusivamente como teoria, não é condição suficiente para uma compreensão adequada do fenómeno, sendo que um importante contributo pode advir da medição efetiva das perceções dos colaboradores, medição essa que permite construir, e em última análise constitui, um valioso auxiliar de tomada de decisões para as organizações. A análise das perceções dos agentes organizacionais sobre o poder efetivamente detido pelos dirigentes máximos, em função de variáveis de caracterização como gênero, idade, antiguidade organizacional ou natureza das funções exerci- 
das, que têm influência direta ou indireta na sua atividade e/ou decisões, permite dar resposta a um conjunto relevante de interrogações.

$\mathrm{O}$ estudo apresentado neste artigo incidiu na variável de medida relativa ao poder detido pelo dirigente máximo de cada organização face aos demais dirigentes da mesma, num contexto posterior à entrada em vigor da Lei n. 66-B/2007, de 28 de dezembro de 2007, e numa das variáveis de caracterização anteriormente mencionadas, o gênero dos colaboradores. Este trabalho avaliou a validade estatística da afirmação de que existem diferenças, ao nível do gênero, nas perceções sobre o poder detido pelos dirigentes máximos dos serviços do Ministério da Justiça em Portugal, sugerindo que é necessário implementar medidas que garantam esse desejável equilíbrio.

\section{Considerações sobre a Forma como a Implementação do SIA- DAP Pode Intensificar as Perceções dos Colaboradores diante dos Dirigentes Máximos das Organizações - A Perspetiva da Teoria Institucional e do Conceito de Ranking}

Sendo um processo que envolve gestão da mudança, é natural que a implementação do SIADAP na administração pública, em geral, e no Ministério da Justiça, em particular, tenha provocado distorções, mais ou menos intensas, na forma como os colaboradores percecionam os dirigentes máximos das organizações. A teoria institucional, coadjuvada pelo conceito de ranking, pode fornecer uma perspetiva sobre a forma como as tensões surgidas neste processo são negociadas por chefias e colaboradores.

$\mathrm{O}$ primeiro passo consiste em reconhecer o SIADAP, per se, à luz das definições avançadas quer por Ostrom, quer por Jepperson (OSTROM, 1990; JEPPERSON, 1991), como padrão social caracterizado por uma sequência de interações-padrão, como uma instituição (CORREIA, 2012).

Feito esse reconhecimento, a teoria institucional pode dar um primeiro contributo, no entendimento da relação colaboradores-SIADAP-chefias, no que diz respeito aos processos de legitimação das instituições. 
A procura de estabilidade em termos de recursos, leva a que as organizações e os indivíduos anseiem por legitimação, que pode ser alcançada por meio do estabelecimento de relações de natureza política, legal, organizacional ou cultural (CORREIA, 2012). Esses processos e tentativas de legitimação, ensaiadas quer por indivíduos quer por organizações, alteram, muitas vezes de forma dramática, a forma como os vários agentes de uma organização se relacionam entre si e percecionam a atividade dos restantes membros.

A título de exemplo, há os processos de implementação tecnológica. Considerando que o SIADAP, enquanto sistema de avaliação de desempenho, pode ser encarado como uma tecnologia ou sistema de informação organizacional, já que é importante perceber que, como tal, ele constitui um poderoso mecanismo de legitimação individual ou organizacional. À luz desse prisma, Fountain (2001) mostra que não existe determinismo tecnológico, mas que as tecnologias e sistemas de informação são implementados por dirigentes e colaboradores, de forma a refletir o envolvimento legal, político, social e a própria cultura organizacional, levando a resultados finais díspares do que seria de esperar na base de uma lógica meramente tecnológica e de eficiência. No que se refere à legitimação por via da implementação tecnológica, é de esperar que dirigentes e serviços com envolventes legais, políticas e sociais distintas, e com culturas organizacionais diversas, tenham levado a cabo processos de implementação da instituição SIADAP (aqui tida como tecnologia e/ou sistema de informação) que conduziram a resultados finais potencialmente bastante diferentes. O mesmo é dizer que a forma como o SIADAP foi implementado em cada serviço do Ministério da Justiça, não sendo determinista, necessariamente influenciou as perceções dos colaboradores que a este passaram a estar sujeitos num conjunto alargado de aspetos e, em particular, relativamente ao poder organizacional detido pelo dirigente máximo da organização face à globalidade da estrutura preexistente.

Um segundo passo, igualmente importante, é reconhecer o SIADAP, dada a sua natureza de sistema de avaliação e monitorização, como um tipo especial de instituição: uma instituição geradora de rankings (CORREIA, 2012). Tendo em conta o enquadramento da teoria institucional, é fundamental analisar com algum detalhe o papel dos rankings 
(listas ordenadas), e as suas consequências, nas organizações. Os efeitos produzidos pelos rankings devem ser tidos em consideração, uma vez que se refletem profundamente nas organizações e nos indivíduos que as constituem.

Foucault numa primeira fase, e Covaleski, mais tarde, descrevem o modo como as pressões de disciplina coerciva se transformam num mecanismo de autogestão (FOUCAULT, 1977; 1980; COVALESKI et al., 1998) que amplifica influências por meio de mudanças nas perceções (cuja aferição foi objetivo do estudo apresentado neste artigo), expectativas e comportamentos dos componentes das organizações.

Existem três grandes aspetos a considerar na estrutura de monitorização que os rankings devem garantir. Desses três aspetos, aquele que merece destaque neste artigo é o da monitorização contínua. Os rankings são usados em última análise para interpretar uma gama díspar de motivações e comportamentos, mesmo que a sua ligação aos rankings seja ambígua. Porém, a vigilância implícita que a divulgação de rankings origina, leva a que até mesmo decisões relativamente triviais sejam reportadas, produzindo um efeito que os dirigentes máximos das organizações descrevem como omnipresente. A reação de serviços e indivíduos ao poder disciplinador dos rankings introduzidos, gerados, e feitos circular pelo SIADAP, é entendida sobretudo como resposta adaptativa de um conjunto de agentes que lutam para reconciliar a sua identidade como profissionais a quem foi imposta uma lógica de responsabilização. Neste sentido a monitorização, vigilância e normalização de procedimentos facilitadores da disciplina proporcionadas pelos rankings do SIADAP transformam não apenas o modo como a sociedade em geral encara a administração pública, como também impõem mudanças à forma como os diretores-gerais e presidentes dos serviços públicos equacionam a sua atividade e as suas relações com os seus pares (serviços e dirigentes) e colaboradores (funcionários). Isso porque, ao fim e ao cabo, a disciplina introduzida pelos rankings fica devendo à instituição SIADAP, que tem força de lei, mas muito do que sucede no processo de implementação e monitorização, principalmente o que corre menos bem ao longo do exercício, acaba por, de um modo ou de outro, ser imputado ao dirigente máximo do serviço, pelos seus colaboradores. Esse aspeto da vivência na esfera das institui- 
ções públicas é adequadamente descrita pela teoria da escolha racional, que refere que as estratégias de maximização de preferências giram em torno da troca de condescendência por primazias, como parte de uma negociação social que, a não ser mantida, pode levar à destruição do padrão de poder institucional existente (LEVI, 1990).

Outros estudos sobre dinâmicas organizacionais demonstram que são três as principais influências que contribuem para a aceitação de atividades inerentemente técnicas como é o caso do SIADAP. A primeira consiste na correspondência entre os objetivos impostos e as identidades e estruturas existentes na organização (ESPELAND, 1998; KALEV et al., 2006; DOBBIN; KELLY, 2007). A segunda envolve o conhecimento de estratégias de desacoplamento anteriormente aplicadas, no sentido de afastamento completo da implementação da política original (WESTPHAL; ZAJAC, 1998; 2001). Por fim, a terceira influência refere-se ao poder detido pelo diretor-geral ou presidente da organização relativamente aos seus diretores (Sauder e Espeland; 2009). Foi exatamente nesta terceira influência que incidiu o foco do estudo descrito neste artigo, tendo o conceito do poder detido pelo dirigente máximo da organização face aos seus diretores, estado na origem da construção da questão efetivamente colocada aos colaboradores do Ministério da Justiça para aferição das suas perceções.

\section{Metodologia}

A componente empírica deste estudo teve início com a inquirição de 458 colaboradores pertencentes a oito serviços do Ministério da Justiça em Portugal, 374 dos quais apresentaram resposta válida quer à variável de caracterização gênero, quer à variável de medida do nível das perceções. O instrumento de recolha de dados, baseou-se no inquérito por questionário. A utilização da fórmula de cálculo da dimensão amostral para proporções, considerando um cenário de variância máxima, um nível de significância de $0,05(5,00 \%)$ e uma dimensão populacional infinita, permitiu quantificar a precisão absoluta do estudo em cerca de 0,0507 $(5,07 \%)$. 
Na variável de caracterização referente ao gênero foram utilizadas as categorias usuais, referindo-se ao gênero de cada colaborador do Ministério da Justiça que participou na investigação:

a) feminino; e

b) masculino.

A variável de medida quantitativa, referente ao nível das perceções dos colaboradores relativamente ao poder detido pelo dirigente máximo da organização, foi criada por Correia (CORREIA, 2012), com base no conceito de distribuição de poder entre a direção (SAUDER; ESPELAND, 2009), e teve a seguinte formulação:

a) Grau em que considero que o dirigente máximo da organização tem poder sobre os restantes dirigentes.

Foi utilizada uma escala por intervalo, numérica, de Likert com 10 pontos, âncoras nos extremos (extremo inferior: "muito baixo"; extremo superior: "muito elevado"), e com a possibilidade de assinalar a opção "não sabe/não responde".

Não foi possível utilizar o teste de igualdade das médias na determinação das relações estatísticas presentes nos dados, uma vez que não se verificou uma das suas condições de aplicabilidade: normalidade da distribuição dos níveis das perceções sobre o poder detido pelo dirigente máximo da organização, em cada categoria de gênero. Por esse motivo, optou-se pela utilização do teste não paramétrico de Mann-Whitney (MANN; WHITNEY, 1947), que foi empregue na determinação da existência, estatisticamente significativa, de valores mais elevados de níveis de perceções para colaboradores do gênero masculino do que para colaboradores do gênero feminino, ou vice-versa. Em qualquer um dos testes anteriormente referidos, o nível de significância utilizado foi de 0,05 $(5,00 \%)$. 

estatísticas de diferenças em função do gênero dos colaboradores?

\section{Resultados}

Das 374 observações, foi apurado que (Tabela 1) 252 correspondiam aos colaboradores do gênero feminino $(67,4 \%)$ e 122 aos colaboradores do gênero masculino $(32,6 \%)$.

Tabela 1: Número de observações por categoria de gênero e respectivo peso percentual

\begin{tabular}{|c|c|c|}
\hline Gênero & Número de observações & Percentagem do total \\
\hline Feminino & 252 & $\mathbf{6 7 , 4 \%}$ \\
\hline Masculino & 122 & $\mathbf{3 2 , 6 \%}$ \\
\hline Total & 374 & $\mathbf{1 0 0 , 0 \%}$ \\
\hline
\end{tabular}

Fonte: Elaborada pelo autor deste artigo

O Gráfico 1 apresenta a distribuição dos resultados obtidos para a variável de medida relativa ao poder detido pelo dirigente máximo de cada organização. Considerando a globalidade das 374 observações válidas, o valor médio apurado para o nível das perceções dos colaboradores relativos a esta variável foi de 6,46 pontos, correspondendo a um nível elevado (entre 5 e 8 pontos).

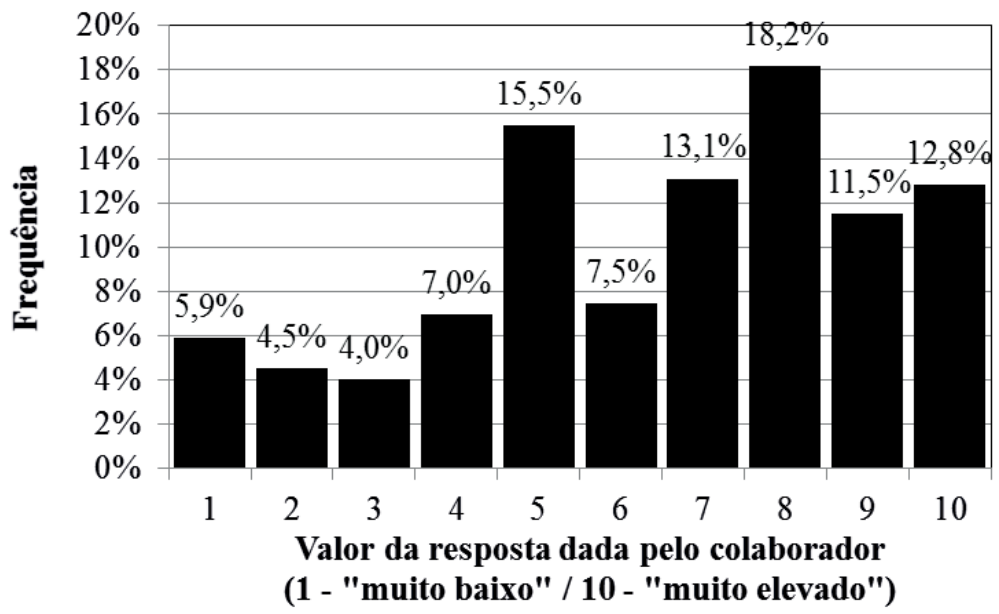

Gráfico 1: Distribuição da variável de medida relativa às percepções dos colaboradores diante do poder detido pelo dirigente máximo de cada organização sobre os restantes dirigentes

Fonte: Elaborado pelo autor deste artigo 
O Gráfico 2 apresenta os valores obtidos para as médias dos níveis de perceção dos colaboradores dos serviços do Ministério da Justiça em Portugal, diante do poder detido pelo dirigente máximo de cada organização sobre os restantes dirigentes, segundo o gênero do respondente. É possível observar que os colaboradores do gênero masculino apresentam um valor médio de 7,05 pontos, 0,87 pontos acima do valor médio dos colaboradores do gênero feminino, que apresentam uma média de 6,18 pontos (ambos correspondendo a níveis elevados). A questão que se coloca é saber se esta diferença observada pode ser atribuída a flutuações aleatórias ou se corresponde a uma diferença de resultados estatisticamente significativa. Para tal, e de acordo com o referido no tópico dedicado à metodologia, foi aplicado o teste não paramétrico de Mann-Whitney.

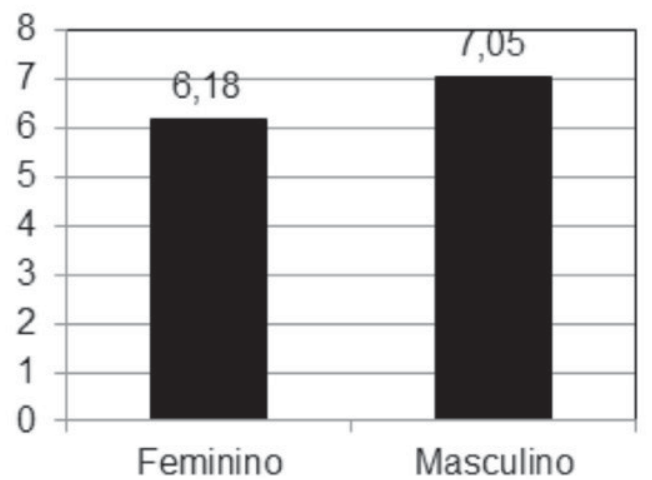

Gráfico 2: Valores dos níveis de percepção dos colaboradores dos serviços do Ministério da Justiça em Portugal, diante do poder detido pelo dirigente máximo de cada organização sobre os restantes dirigentes, segundo o gênero Fonte: Elaborado pelo autor deste artigo

Detalhes sobre as distribuições da variável de medida para indivíduos do gênero feminino e masculino são particularizados no Gráfico 3. A Tabela 2 apresenta os resultados obtidos por intermédio da aplicação do teste não para métrico de Mann-Whitney. Destaca-se em particular o $p$-valor obtido, inferior a 0,000. Uma vez que o nível de significância definido para a partida $(0,05)$ é superior ao $p$-valor, existem evidências estatísticas que conduzam à rejeição da hipótese nula do teste, ou seja, existem evidências estatísticas de valores mais elevados de níveis de perceção 
sobre o poder detido pelo dirigente máximo de cada organização sobre os restantes dirigentes, em colaboradores dos serviços do Ministério da Justiça do gênero masculino do que em colaboradores dos serviços do Ministério da Justiça do gênero feminino, em Portugal.

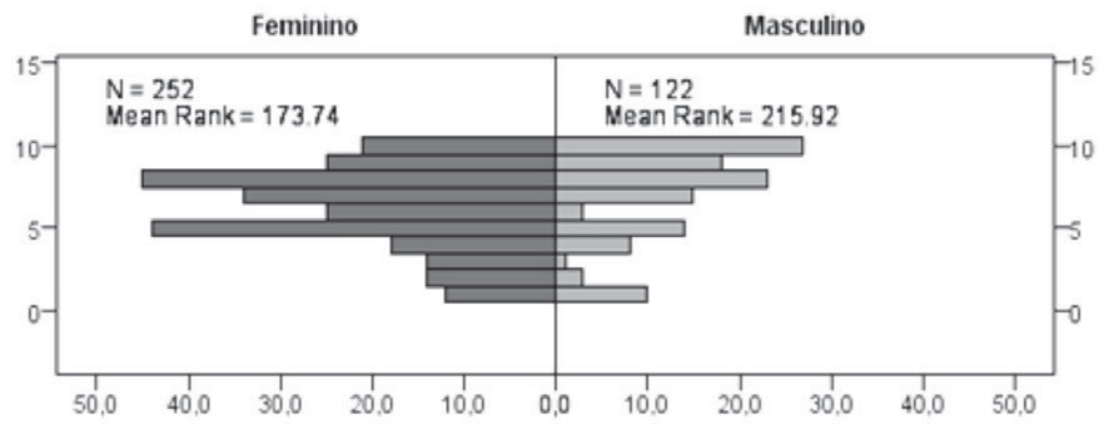

Gráfico 3: Distribuição de frequências da variável de medida relativa às percepções dos colaboradores diante do poder detido pelo dirigente máximo de cada organização sobre os restantes dirigentes, segundo o gênero Fonte: Elaborado pelo autor deste artigo

Tabela 2: Resultados do teste de Mann-Whitney para a variável de medida relativa às percepções dos colaboradores face poder detido pelo dirigente máximo de cada organização sobre os restantes dirigentes, agrupada segundo o gênero

\begin{tabular}{|c|c|}
\hline Valor do teste MANN-WHITNEY & 11904,500 \\
\hline Valor de Z & -3.568 \\
\hline$p$-valor (bicaudal) & 0.000 \\
\hline
\end{tabular}

Fonte: Elaborada pelo autor deste artigo

\section{Conclusão}

As relações encontradas nos dados referentes ao estudo tratado neste artigo apontam os colaboradores do gênero masculino dos serviços do Ministério da Justiça em Portugal como apresentando valores de níveis de perceção, acerca do poder detido pelo dirigente máximo da sua organização sobre os restantes dirigentes, significativamente mais elevados do que os colaboradores do gênero feminino. 
Efetivamente, na literatura, é possível encontrar estudos que destacam a existência de expectativas distintas para com a atividade laboral em função do gênero (GRUNEBERG, 1979). Essas expectativas se concretizam, por exemplo, numa maior valorização das oportunidade de influenciar decisões importantes e dirigir o trabalho de outros colaboradores na organização por parte dos homens, enquanto as mulheres tendem a encarar a possibilidade de executar tarefas que contribuam para a sua realização pessoal e as relações com os colegas como fatores mais relevantes (SCHULER, 1975). Simultaneamente, os homens tendem a sentir-se mais satisfeitos com as chefias que as mulheres (ZAWACKI et al., 1995), e esse facto pode, por si só, explicar uma parte considerável do fenômeno descrito neste estudo. Se os homens tendem a sentir-se mais satisfeitos com as chefias que as mulheres, então, provavelmente serão mais propensos a considerar essas mesmas chefias mais competentes detentoras de maior poder organizacional.

Bastantes outros estudos, levados a cabo numa série de ambientes de trabalho e atividades profissionais distintas, salientam que, em regra, os indivíduos do gênero feminino exercem funções em que as oportunidades de progressão na carreira, níveis de autoridade, status e remuneração são inferiores (HULL, 1999; ROUT, 1999; JOHNSON; JOHNSON, 2000), o que, a verificar-se poderia afetar a forma como as colaboradoras das organizações percecionam o poder dos dirigentes máximos. A introdução e implementação de um sistema de avaliação de desempenho laboral como o SIADAP, se viesse a demonstrar ser um sistema intensificador de desigualdade e discriminação de gênero, poderia revelar-se como um dos vetores deste conjunto de fenómenos multidimensional.

Porém, no caso dos serviços do Ministério da Justiça em Portugal, não existem evidências que permitam conjeturar nesse sentido, não apenas porque, por força de lei, a igualdade dos níveis remuneratórios e de oportunidades de progressão na carreira para os dois gêneros está assegurada, mas também porque a proporção de colaboradores do gênero feminino está em maioria, estando próximo de um rácio de três para um, face aos colaboradores do gênero masculino, abundando exemplos de chefias intermédias e dirigentes máximos de serviços do gênero feminino, dotadas de legítimo status, autoridade e poder organizacional. Recorde-se 
que, para a data da realização deste artigo, a própria Ministra da Justiça em Portugal é uma mulher.

Por isso mesmo, os factos descritos nos estudos de Hull, Rout ou Johnson e Johnson (HULL, 1999; ROUT, 1999; JOHNSON; JOHNSON, 2000) não podem ajudar a explicar a existência de diferenças estatisticamente significativas entre os níveis de perceção dos indivíduos do sexo masculino e feminino, nos serviços do Ministério da Justiça, face ao poder detido pelo dirigente máximo da sua organização sobre os restantes dirigentes (os indivíduos do sexo feminino parecem não reconhecer aos dirigentes máximos tanto poder como aquele que lhes é reconhecido pelos indivíduos do gênero masculino). A explicação do fenômeno aqui reportado parece estar mais próxima das constatações anteriormente referidas, feitas por Gruneberg, Schuler ou Zawacki (SCHULER, 1975; GRUNEBERG, 1979; ZAWACKI et al., 1995) e, eventualmente, mais próxima de diferenças a nível da constituição bioquímica e psicológica de cada gênero, que poderão, por ser turno, justificar uma maior propensão do gênero masculino no reconhecimento e aceitação de figuras de autoridade. Trabalhos futuros sobre esta temática deverão tentar esclarecer os fenômenos, efetivamente em causa, que subjazem à diferença de perceções aqui reportada, entre os gêneros.

Adicionalmente, esses resultados fornecem mais uma peça, a juntar ao conjunto cada vez mais avassalador de resultados já existentes, que contribui para o desmoronar do paradoxo segundo o qual as mulheres apresentam níveis de perceção mais elevados acerca das variáveis relacionadas com a atividade profissional, fenómeno também conhecido genericamente como paradox of the contented working woman (TOLBERT; MOEN, 1998. Nesta pesquisa, em particular, o contributo é dado ao nível de evidências que se reportam à variável laboral, teórico-institucional, poder detido pelo dirigente máximo da organização sobre os restantes dos dirigentes. Os resultados obtidos facultam ainda a possibilidade de conjeturar não só a continuação, no século XXI, da tendência reportada por Souza-Poza e Souza-Poza para o nivelamento das perceções laborais de mulheres e homens, registrada ao longo de 1990 (SOUZA-POZA; SOUZA-POZA, 2003), mas até, eventualmente, a inversão dessa tendência, com indivíduos do gênero masculino que apresentam, de forma consis- 
tente e continuada, valores de percepções mais elevados do que indivíduos do gênero feminino, nas próximas décadas do século XXI. O impacto, reportado por Correia (2002), das perceções dos colaboradores sobre o SIADAP na satisfação, lealdade e envolvimento laborais, com todas as consequências que daí advêm, contribui com um ponto adicional de interesse para que esta temática continue sob observação, neste início de século.

Estudos futuros, no atual e renovado contexto posterior à entrada em vigor da Lei n. 66-B/2007, de 28 de dezembro, deverão procurar testar a validade desses resultados não apenas no setor da justiça, nem apenas noutros setores de atividade em Portugal, mas também em contextos públicos e privados noutras regiões geográficas, de modo a tornar as conclusões mais robustas e abrangentes. Sugere-se ainda o estudo dos perfis de perceções dos colaboradores acerca do poder detido pelo dirigente máximo sobre os restantes dirigentes, na organização onde trabalham, segundo outras variáveis de caracterização organizacional e sociodemográfica que possam ser fatores de desigualdade e de discriminação, como sejam a natureza das funções exercidas, a antiguidade organizacional ou a idade dos colaboradores.

\section{Referências}

CORREIA, Pedro. Sobre o SIADAP, a Teoria Institucional e o Ministério da Justiça em Portugal. Scientia Iuridica - Revista de Direito

Comparado, [S.l.], v. 60, n. 325, p. 101-130, 2011.

CORREIA, Pedro. O Impacto do Sistema Integrado de Gestão e Avaliação do Desempenho da Administração Pública (SIADAP) na Satisfação dos Colaboradores: o Caso dos Serviços do Ministério da Justiça em Portugal. 2012. 549 p. Tese (Doutorado em Ciências Sociais) - Especialização de Administração Pública. Universidade Técnica de Lisboa, 2012. 

estatísticas de diferenças em função do gênero dos colaboradores?

COVALESKI, Mark et al. The Calculated and the Avowed: Techniques of Discipline and Struggles over Identity in Big Six Public Accounting Firms. Administrative Science Quarterly, [S.l.], v. 43, n. 293-327, 1998.

DOBBIN, Frank; KELLY, Erin. How to Stop Harassment: The Professional Construction of Legal Compliance in Organizations. American Journal of Sociology, [S.l.], v. 112, n. 4, p. 1.203-1.243, 2007.

ESPELAND, Wendy. The Struggle for Water: Politics, Rationality and Identity in the American Southwest. Chicago, IL: University of Chicago Press, 1998.

FOUCAULT, Michel. Discipline and Punish: The Birth of the Prison, London, UK: Allen Lane, 1977.

FOUCAULT, Michel. Power/Knowledge: Selected Interviews and Other Writings 1972-1977, Gordon, Colin (Ed.). New York: Pantheon, 1980.

FOUNTAIN, Jane. Building the Virtual State: Information Technology and Institutional Change. Washington, DC: Brookings Institution Press, 2001.

GRUNEBERG, Michael. Understanding Job Satisfaction. London: MacMillan and Co., 1979.

JEPPERSON, Ronald. Institutions, Institutional Effects, and Institutionalism. In: POWELL, Walter; DIMAGGIO, Paul (Ed.). The New Institutionalism in Organizational Analysis. Chicago: University of Chicago Press, 1991. p. 143-163.

HULL, Kathleen. The Paradox of the Contented Female Lawyer. Law \& Society Review, [S.l.], v. 33, n. 3, p. 687-703, 1999.

JOHNSON, Gloria; JOHNSON, W. Roy.Perceived Overqualification and Dimensions of Job Satisfaction: A Longitudinal Analysis. Journal of Psychology, [S.l.], v. 34, n. 5, p. 537-556, 2000. 
KALEV, Alexandra et al. Best Practices or Best Guesses? Assessing the Efficacy of Corporate Affirmative Action and Diversity Policies. American Sociological Review, [S.l.], v. 71, p. 589-617, 2006.

LEVI, Margaret. A Logic of Institutional Change. In: COOK, Karen; LEVI, Margaret (Ed.). The Limits of Rationality. Chicago: University of Chicago Press, 1990. p. 401-418.

MANN, Henry; WHITNEY, Donald. On a Test of Whether One of Two Random Variables is Stochastically Larger than the Other. Annals of Mathematical Statistics, [S.l.], v. 18, n. 1, p. 50-60, 1947.

OSTROM, Elinor. Governing the Commons: The Revolution of Institutions for Collective Action. Cambridge: Cambridge University Press, 1990.

PORTUGAL. Lei n. 66-B/2007, de 28 de dezembro. Diário da República, de 28 de dezembro de 2007. Lisboa, Portugal, [2007].

ROUT, Usha. Gender Differences in the Stress, Satisfaction and Mental Well-Being among General Practitioners in England. Psychology, Health \& Medicine, [S.l.], v. 4, n. 4, p. 345-355, 1999.

SAUDER, Michael; ESPELAND, Wendy. The Discipline of Rankings: Tight Coupling and Organizational Change. American Sociological Review, [S.l.], v. 74, n. 2, p. 63-82, 2009.

SCHULER, Randall. Sex, Organization Level and Outcome Importance: Where the Differences Are? Personnel Psychology, [S.l.], v. 28, p. 365375, 1975.

SOUSA-POZA, Alfonso; SOUSA-POZA, Andrés. Gender Differences in Job Satisfaction in Great Britain, 1991-2000: Permanent or Transitory? Applied Economics Letters, [S.l.], v. 10, n. 11, p. 691-694, 2003.

TOLBERT, Pamela; MOEN, Phyllis. Men's and Women's Definitions of "Good" Jobs". Work \& Occupations, [S.l.], v. 25, n. 2, p. 168-195, 1998. 
WESTPHAL, James; ZAJAC, Edward. Symbolic Management of Stockholders: Corporate Governance Reforms and Shareholder Reactions. Administrative Science Quarterly, [S.l.], v. 43, p. 127-153, 1998.

WESTPHAL, James; ZAJAC, Edward. Decoupling Policy from Practice: The Case of Stock Repurchase Programs. Administrative Science Quarterly, [S.l.], v. 46, p. 202-228, 2001.

ZAWACKI, Robert et al. Who Has Higher Job Satisfaction. Male or Female Nurses? Nursing Management, [S.l.], v. 26, n. 1, p. 54-55, 1995.

Pedro Miguel Alves Ribeiro Correia é professor de administração pública e análise de dados no Instituto Superior de Ciências Sociais e Políticas da Universidade de Lisboa (Portugal) e doutor em Ciências Sociais com ênfase em Administração Pública pela Universidade Técnica de Lisboa (Portugal). É ainda investigador integrado do Centro de Administração e Políticas Públicas (CAPP), investigador colcaborador do Centro Interdisciplinar de Estudos de Gênero (CIEG), Coordenador do Observatório Nacional de Administração Pública (ONAP) e Consultor da Direção-Geral da Política de Justiça do Ministério da Justiça de Portugal.

E-mail: pcorreia@iscsp.ulisboa.pt.

Endereço profissional: Universidade Técnica de Lisboa, Instituto Superior de Ciências Sociais e Políticas, Centro de Administração e Políticas Públicas, Rua Almerindo Lessa, Lisboa, Portugal -1300-663. 